By acceptance of this articte, the publisher or racipienc acknowlydges the US. Governmant's right to retain ononexcluzivn, royalty-iree lleense in and to any convight cowering the ar ticlo.

\title{
REACTOR ASSESSMENTS OF ADVANCED BUMPY TORUS CONFIGURATIDNS*
}

\author{
N. A. Uckan, L. H. Owen, and D. A. Spong \\ Oak Ridge National Laboratory \\ Oak Ridge, Tennessee 37831 \\ R. L. Miller \\ Applied Microwave Plasma Concepts. Ine. \\ Encinitas, California 92024
}

W. B. Ard, J. F. Pipkins, and R. J. Schmitt McDonnell Douglas Astronautics Company

St. Louis, Missouri 63166

\section{MOTICE \\ PORTOUS OF THIS REPORT ARE ILLEGIBLE. \\ it has been reproduced from the best avallable copy to permit the broadest possible availability.}

\footnotetext{
*Research sponsored by the Office of Fusion Energy, U. S. Department of Energy, under Contract No. W-7405eng-28 with Union Carbide Corporation and under Contract No. DE-ACO3-82ER51030 with AMPC, Inc.
}

\section{DISCLAIMER}

This report was prepared as an account of work sponeored by an agency of the United States Government. Neither the United States Government nor any agency thereof, nor any of their employees, makes any warranty, express or implied, or asumes any kegal liability or responsbility for the accuracy, completeness, or usefulness of any information, apparatus, product, or process disclosed, or represents that its uve would not infringe privately owned rights. Reference herein to any specific commercial product, process, or service by trade name, trademart, manufacturer, or otherwive does not necesurily contitute or imply its exdorsement, secommendation, of fuvoring by the Unitod States Government of any atency thereof. The view and opinions of authors expresed herein do not necesearily state of selloct thone of the United States Government or any agency thereor. 
Recently, several configurational approaches aild concept improvement schemes were introduced for enhancing the performance of the basic ELMO Bumpy Torus (EBT) concept and for improving its reactor potential. These configurations include planar racetrack and square geometries, Andreoletti coil systems, and bumpy torus-stellarator hybrids (which include twisted racetrack and helical axis stellarator-snakey torus). Preliminary evaluations of reactor implications of each of these configurations have been carried out based on magnetics (vacuum) calculations, transport and scaling relationships, and stability properties. Results indicate favorable reactor projections with a significant reduction in reactor physical size as compared to conventional EBT reactor designs carried out in the past. Specifically, with these advanced configurations, reactors with $R \simeq 20 \pm 3 \mathrm{~m}$ are found to be possible; this is almost a factor of 2 reduction in size compared to recent EBT reactor design points with $R \simeq 40 \pm 5 \mathrm{~m}$ (which utilizes symmetrizing coils for an aspect ratio enhancement) for comparable power outputs. (Here $R$ is defined as $2 \pi R$-equivalent major radius.) This makes it possible for operating at high wall loadings $\left(\sim 2-4 \mathrm{mH} / \mathrm{m}^{2}\right.$ instead of $\left.\sim 1-1.5 \mathrm{MH} / \mathrm{m}^{2}\right)$ and increased engineering fusion power density.

\section{Introduction}

Several aspects of the ELMD Bumpy Torus (EBT) concept make it attractive as a fusion reactor: the large aspect ratio; simple, noninterlocking circular coils with modest field; modularity; and, above all, the steady-state operation. There have been several EBT reactor studies, with the most recent one discussed in Ref. [1]. Conventional EBT designs have raised two issues that can be considered as disadvantages for a 
reactor. The first is associated with the large unit size. This has to do with the bulk plasma confinement properties, which are closely related to the dependence of the particle drift orbits on pitch angle. Relatively large aspect ratio $\left(R_{T} / R_{c}\right.$, where $R_{T}$ is the major radius of the torus and $R_{c}$ is the mean coil radius $\simeq$ radius of curvature) is required to attain large $n \tau$. The second is the recirculating power required to maintain the energetic hot electrons (hot electron rings), required for bulk plasma stability.

During the r-cent U.S.-Japan Workshop on Advanced Bumpy Torus Concepts, several configurational approaches and concept improvement schemes emerged for enhancing the performance of the basic EBT concept and for improving its reactor potential [2]. These include (but are not limited to) configurations with (1) noncircular magnetic coils-Andreoletti coil systems [3]; (2) square or racetrack geometries without [4] or with [5] rotational transform; and (3) stellarator-bumpy torus hybrids - snakey torus [6]. In all cases EBT-like hot electron rings are usad to stabilize the interchange modes driven by the unfavorable magnetic field curvature either on a continuous, steady-state basis [for cases (1) and (2) above] or on a transient basis during startup to access the "second stability regime" [for case (3) above]. In addition to these new advanced configurations, concept improvements such as positive ambipclar potential operation and various radiofrequency and microwave heating scenarios for profile control and tailored stability properties offer the possibility of enhanced performance in the present EBT geometry (see Ref. [2]). 
These configurations, basically, fall into two categories: (1) closed field line devices (such as planar racetrack and square geometries and Andreoletti coil systems) and (2) closed flux surface devices [such as twisted racetrack (TRT) and snakey torus]. Closed field line devices rely on the "bumpiness" of the field (grad-B, as well as radial electric field $E_{r}$ ) for closed drift surfaces and equilibrium and require hot electron rings (in bad curvature regions) for stability. These are the properties of EBT. Closed flux surface (a property not found in EBT) geometries can further be divided into two categories: those using rotational transiorm in bumpy tori to improve the confinement (bumpy torus with stellarator features, such as a TRT) and those using some of the EBT features (hot electrons) in closed flux surface devices to enhance the stability during startup (e.g., a stellarator with EBT features, such as a snakey torus).

This paper summarizes the results of recent preliminary reactor assessments of these advanced bumpy torus configurations. Included in the analysis are magnetics (vacuum) calculations, confinement scaling, and stability properties. The technology requirements and engineering characteristics are only considered to the extent that they are largely different from those in conventional EBT reactors (or other fusion reactors).

\section{Comments on Basic EBT Configuration}

The design of an EBT experiment and/or reactor requires determination of the best magnetic field configuration to maximize vacuum field particle confinement within the toroidal volume. The toroidal curvature of the magnetic field in EBT results in an inward shift of particle drift orbits toward the major axis. The relative amount of shift, however, depends on 
the pitch angle $\left(V_{\|} N\right)$, and this shift is largest for the transitional (resonant) and toroidally passing particles (i.e., those having large $V_{\|} / N$ ). This displacement of particle drift orbits plays a major role in diffusive and direct particle losses.

The commonly used criteria for the assessment of EBT magnetic geometries are based on the vacuum magnetic field drift orbits and the volumetric efficiency. The drift orbits are determined by contours of constant $J$, the longitudinal adiabatic invariant $\left(J=\oint V_{\|} d \ell\right)$. The shift in drift crbit centers is then determined from $R_{J M I N}$, the minimum of $J$. The volumetric efficiency $F\left(V_{\|} / N\right)=A\left(V_{\|} / N\right) / A\left(V_{\|}=0\right)$ is defined as the ratio of the last closed drift orbit area of a particle with a given pitch angle to that of $V_{\|}=0$ (purely trapped particle). The centering of drift orbits for deeply trapped particles within the vacuum chamber is important for the utilization of the magnetic volume because it determines the spatial position of the hot electron rings, which determines the boundary of a toroidally confined core plasma. (Note that the rings form near contours of constant mod- $B$ in the midplane.)

In a simple bumpy torus, an increase in aspect ratio reduces the dispersion in drift orbits and improves the confinement of all classes of particles. The need for better confinement characteristics and efficient utilization of magnetic fields has led in the past to reactors with large physical size and power output. Through the use of low-current supplementary coils (i.e., ARE and SYM coils [7]) or inverse-D coils [1], however, it was possible to reduce the reactor sizes from about $R_{T} \simeq 60 \mathrm{~m}$ [8] to about $R_{T} \simeq 35 \mathrm{~m}$ [1] for a fusion power output of $P \sim 1200-1500$ Whe. Results of the recent reactor studies with SYM and inverse-D coils are given in Ref. [1]. With the new advanced configurations, further reduction in reactor physical 
size has been shown to be possible. He discuss their characteristics in the following sections. In ail cases, unless otherwise specified, the following parameters are used: average plasma radius $\bar{a} \simeq 1.5 \mathrm{~m}$ $\left(a=1 \mathrm{~m}\right.$ under the coil); $B_{\infty}=B_{\min } \simeq 2.5 \mathrm{~T}$ and $B_{\max } \simeq 5.5 \mathrm{~T}$ for mirror fields on-axis; the blanket and shield thickness under the mirror coil $\simeq 1.0 \mathrm{~m}$ and is distributed nonuniformly between the coils; and the mirror coil half thickness $\simeq 0.35 \mathrm{~m}$.

\section{EBT with Andreoletti Coils (EBTEC)}

EBTEC (EBT with enhanced confinement) configuration consists of a toroidal array of racetrack-shaped coils - "Andreoletti coils" - whose major axes are alternately oriented vertically and horizontally [3]. By adjusting the elongation of the coils $(\mathrm{H} / \mathrm{H}$, heightto-width ratio) and the relative shifts in the center of alternate coils, it has been shown that it is possible to obtain nearly concentric trapped and passing particle drift surfaces, thus greatly reducing the random-walk step size for diffusion. For a next-generation-size device (such as EBT-P). the EBTEC configuration, at large aspect ratio, shows closed and nearly circular resonant particle drift orbits [3]. As in EBT, the core plasma is stabilized by the hot electron rings which form near mod-B contours in the midplane. Here we present prel iminary results for a reactor-size device.

For a reactor, we must include the effects of a blanket and shielding, which for a fixed geometry will reduse the plasma radius. To recover a desired plasma radius at fixed major radius $R_{T}$ and mirror ratio $M$, we must increase the coil size and decrease the number of sectors N. A smaller aspect ratio results, and some outer drift surfaces are scraped off by the shielding. 
Among the various sizes studied for a reactor, we present two cases here: (1) greatly enhanced confinement for a device comparable in size to the latest circular coil EBT reactor design, which uses SYM coils for aspect ratio enhancement [1], that is, for $R_{T} \simeq 35 \mathrm{~m}$, and (2): reduced major radius device, $R_{T} \simeq 20-25 \mathrm{~m}$, with confinement comparable to a circular coil reactor $\left(R_{T} \simeq 35 \mathrm{~m}\right)$. In the calculations, blanket and shield thickness is assumed to be $1.0 \mathrm{~m}$ at the limiting coil throat in the shortest direction and nonuniform $(>1 \mathrm{~m})$ distribution of the blanket between the coils (as well as under the coil in the longest direction).

For the enhanced confinement ase $\left(R_{T} \simeq 35 \mathrm{~m}\right.$, $M=2.25$, and $a \simeq 1.25 \mathrm{~m}$ under the coil, Fig. 1 shows the dispersion in drift orbit centers of trapped and passing particles, $\quad \Delta R_{J M I N}=R_{J M I N}\left(V_{\|} / N=0\right)-$ $R_{J M I N}\left(V_{\|} / N=1\right)$, versus the outward shift of the vertical coils relative to the horizontal coils. Because of less shift requirement, $H / N=3$ has been chosen for the coils. To have $\Delta R_{J M I N} \simeq 0$, about a $7.5-m$ shift of coil centers is required. One quadrant of the equatorial plane of the configuration for this case is shown in Fig. 2.

A plot of volumetric efficiency (percent of area enclosed by a drift surface) in the midplane versus $V_{\|} N$ is shown in Fig. 3 for an EBTEC reactor and for a circular coil EBT reactor without and with SYM coils ior the same $R$, $a$, and $M$. Note that the EBTEC has nearly concentric orbits for all $V_{\|} / N$; however, the area for the resonance particles is small due to shielding scrapeoff. It may be possible to alleviate this scrapeoff by proper shaping or parameter optimization. In any case, a very significant improvement in resonance particle behavior over the circular coil case is evident from Fig. 3. 
For the reduced major radius case $\left(R_{T} \simeq 20-25 \mathrm{~m}\right)$. Fig. 4 (a plot of $\Delta R_{J M I N}$ versus $R_{T}$ ) indicates that drift orbit centering becomes poorer as $R_{T}$ decreases. The coil shift is about $10 \mathrm{~m}$; however, a larger shift could be used to reduce $\Delta R_{\text {JMIN }}$ further.

Figure 5 shows a 14-sector device with $R_{T}=28.5 \mathrm{~m}$ for the vertical coils and $R_{T}=16.5 \mathrm{~m}$ for the horizontal coils. The plasma in the midplane is centered at $R_{T}=23 \mathrm{~m}$. Figure 6 shows the limiting field lines in the equatorial plane and the mod- $B$ contours. Note that the distortion from the shift has moved the minimum in mod-B contours of $f$ of the midplane. Nevertheless, a drift surface analysis was carried out in the "geometric midplane." Figure 7 shows the trapped and passing particle drift orbits in this midplane. $\Delta R_{J M I N} \simeq 0.6 \mathrm{~m}$ is lower than the value deduced from Fig. 4, but the plasma radius a is smaller here. Yolumetric efficiency corresponding to this case is also shown in Fig. 3. Comparison with the circular coil EBT reactor still indicates significant improvement.

To summarize the results, we see that for the $R_{T} \simeq 35 \mathrm{~m}$ case, trapped and passing particle orbits can be made to nearly coincide. A very narrow resonance region results (see Fig. 3 ), which can perhaps be eliminated with further optimization; in particular, if $R_{T}$ is increased, the resonant particles become circular at large plasma radius. For $R_{T}=23 \mathrm{~m}$, the $14-c o i l$ case, the confinement is significantly better than for the $R_{T}=35 \mathrm{~m}$ circular reactor. Due to high distortions for small $N$ (see Fig. 6), an analysis away from geometric midplane needs to be developed to accurately assess confinement. 
Perhaps the greatest engineering problem that can be raised with respect to the EBTEC configuration is the large size of the coils $(H / H=3)$. It may, however, be possible to design alternative, smaller coils which produce the same magnetic field geometry as the shifted Andreoletti coils. Coil design using this approach has been done at Garching for stellarators.

\section{ELMO Bumpy Square}

The ELMO Bumpy Polygon geometry in which the magnetic axis is not circular but is shaped like a racetrack, triangle, square, pentagon, etc., consists of linear segments of simple mirrors (made of circular or elliptical coils) that are linked by sections of high-field toroidal solenoids [4]. The ELMO Bumpy Square (EBS) is the member of this class that has been most extensively analyzed. In these configurations, the toroidal effects are localized in regions of high magnetic field, thereby minimizing the effect of toroidal curvature on single particle drift orbits, volumetric efficiency, etc. As in EBT, the core plasma is stabilized by the presence of hot electron rings in the linear mirror sections. For a near-term, experimental-size device (such as EBT-S), EBS has been shown to have single particle confinement properties and plasma volume utilization that are distinctly superior to those of a standard EBT of comparable size [4].

High-field solenoid sections (corners) can be circular or elliptical in cross section; however, an elliptical shape is found to give the best passing particle confinement. It is also possible to replace the coils in the straight sections with the Andreoletti coils discussed previously. Reactor characteristics of the bumpy square (as well as the racetrack) are analyzed for both circular and Andreoletti coils. Significant 
improvements (similar to the results discussed in the previous section) in orbit centering and volumetric efficiency over those of circular coil EBT are found.

Figure 8 shows the variation of the rormalized. magnetic field strength as a function of arc length along the magnetic axis for one quadrant (straight section plus two half-corners) of a typical reactor case, which consists of three linear mirror sectors per side (total of 12 mirrors plus 4 corners). The on-axis mirror ratio in the sides is $\sim 2.2$ and the "global" mirror ratio ( $B$ at the corners/ $B$ at the reference midplane, $B_{00}$ ) for this particular case is 3.6 . The mirror coils on the sides are 8-T magnets, and the corner coils are 12-T. From the shape of the curve in Fig. 8, one can see that there are mirror-trapped particles in a single sector, mirror-trapped particles between the high-field corners, and passing particles. There are also transitional particles that turn or barely pass near the various field maxima. The equivalent major radius of the device (circumference/ $2 \pi$ ) shown in Fig. 8 is about $R_{T} \simeq 20.5 \mathrm{~m}$, which has drift orbit characteristics much better than the $R_{T}=35 \mathrm{~m}$ circular coil EBT (Fig. 3). Transitional and passing particle orbit characteristics are similar to and the trapped particle orbits are significantly better than the $R_{T}=35 \mathrm{~m}$ EBT with SYM coils.

Simple scaling calculations indicate that diffusive step-size $\Delta x\left(=\Delta R_{J M I N}\right)$ is inversely proportional to the global mirror ratio ( $B_{\text {corner }} / B_{0 o}$ ) and the ratio of the length of the straight section to the curved section,

$$
\Delta x \propto\left(\frac{B_{\text {corner }}}{B_{\infty}}\right)^{-1}\left(\frac{N L_{m}}{2 \pi R \text { cor }}\right)^{-1} \times\left(\frac{B_{\text {corner }}}{B_{\infty}}\right)^{-1}\left(\frac{N}{2 \pi}\right)^{-1}
$$


where $\mathrm{N}$ is the number of mirrors (in straight sections). $L_{m}$ is the mirror sector length, and $R_{c o r}$ is the radius of the corner (toroidal solenoid) in which $R_{\text {cor }} \simeq(1-1.2) 4$. He note that confinement time scales with $1 /(\Delta x)^{2}$, thus with the square of the global mirror ratio.

Figure 9 shows a field line drawing (in the equatorial plane) of a hybrid configuration; bumpy square with Andreoletti coils. Here each straight section (side) consists of two mirror sectors with one horizontal Andreoletti coil $(H / W=2)$. Among the configurations studied, this one, (though not optimized) gives the smallest bumpy square geometry for a reactor with an equivalent major radius of $R_{T}=17.5 \mathrm{~m}$. The volumetric efficiency and drift orbit characteristics are much better than the $R_{T}=20.5 \mathrm{~m}$ bumpy square case discussed previously.

Figure 10 shows a field line drawing of an another hybrid configuràtion; bumpy square with horizontal "tear drop" coils in which the shape of the coil is shown in Fig. 11. This is similar to the case discussed previously (see Fig. 9), however, in this case the plasma accupies a larger fraction of the coil volume. The tear drop coils (one on each side) are shifted inward roiative to the corners. Figure 12 shows the variation of the normalized magnetic field strength as a function of arc length along the magnetic axis for one quad:ant, where $B_{\infty} \simeq 2.2 \mathrm{~T}, L_{m} \simeq 10 \mathrm{~m}, \quad$ and $\hat{\mathrm{K}}_{\text {cor }} \simeq 6.5 \mathrm{~m}$. The global mirror ratio is about 5 . The tear drop coils are 10-T magnets with modest current density and the corner coils are 15-T. The corner coils are of a much smaller cross section and the total weight of all the coils in one corner is comparable to that of one tear drop coil. A plot of volumetric efficiency is given in Fig. 13, which compares very favorably with Fig. 3. 


$$
-12-
$$

The concerns that can be raised with respect to the square (or racetrack) configuration are mainly associated with the high-field corners. Although magnetic fields considered for corners are not too high, the cost of these magnets compared to the overall plant cost needs to be analyzed. Because there are a finite number of coils that can be placed in corners, field ripple effects may play an important role. Ballooning modes associated with the corners and the effect of parallel currents on equilibrium and confinement properties are prusently under consideration. The EBT, as a closed field line device, is sensitive to field errors. These field errors may be more siringent in the EBS configuration than in the standard EBT because of the fewer degrees of symmetry.

\section{Twisted Racetrack}

The twisted racetrack (TRT) EBT is a type of "figure-8" stellarator with two straight sections composed of axisymmetric mirror sectors. In this configuration (Fig. 14), as in EBT, the "bumpiness" of the field provides favorable poloidal drifts and equilibrium, and the hot electron rings (in the straight sections) provide the stability. Rotational transform is introduced (by twisting the racetrack) to improve the confinement. The curved solenoid sections do not contain rings (as in the planar bumpy racetrack or bumpy square configurations) and have an on-axis field approximately equal to the magnetic field in the coil throats of straight sections. The flux surfaces are nearly concentric circles with essentially no shear. Thus the objective of TRT is to obtain a configuration with favorable EBT stability and equilibrium properties that has the stellarator-like favorable transport at modest aspect ratio. 
Preliminary calculations carried out for a TRT reactor also indicate considerable improvement (similar to EBTEC and EBS) over a standard EBT configuration. The example looked at is for $R_{T} \simeq 20 \mathrm{~m}$, equivalent circumference major radius. It consists of six axisymmetric mirrors per straight section (with a mirror ratio of $M=2.25$ and mirror length $L_{m} \simeq 6.5 \mathrm{~m}$ ) -a total of 12 mirrors - and curved solenoid end connectors with a radius of $R_{\text {cor }} \simeq 8 \mathrm{~m}$. The rotational transform is $q$ (axis) $\simeq 1.7$ and $q$ (limiter) $\simeq 2.3$.

At reactor temperatures $\left(T_{i} \sim 20 \mathrm{keV}\right)$ the ion collision frequency is lower than the bounce time for ions trapped in the $1 / R$ variation in the toroidal curved sections of the racetrack. In this regime the dominant thermal conductivity is due to these trapped ions, which are in banana orbits. The diffusion coefficient is given roughly by

$$
D \sim v_{g O} \rho_{i}^{2}\left(\frac{q^{2}}{\epsilon} f_{T} \nu^{*}\right)
$$

where $q p_{i} / \epsilon^{1 / 2}$ is the banana width, $f_{T}$ is the fraction of distribution trapped in the banana orbits, $\nu^{*} \nu_{90}$ is the effective collision frequency for untrapping the ions, and $\epsilon=a / R_{\text {cor }}$ is the inverse aspect ratio. The banana width in the TRT is the same as in a tokamak or stellarator with the same toroidal curvature. However, the fraction of trapped particles and the effective collision frequency in the straight section with mirrors are different from those in the toroidal section. Therefore, to evaluate the transport in TRT, the parameters must be integrated around the device. Calculations are carried out by approximating the mirror sectors as square magnetic well with a field $B_{\min }=B_{\max } / M$ for half the length of the sector $\left(L_{m} / 2\right)$ and $y$ field $B_{\max }$ for the other half of the length. For 
a density of $\sim 10^{20} \mathrm{~m}^{-3}$, a temperature of $\sim 20 \mathrm{keV}$, and $q \simeq 1.7$, we find $n T \simeq 3.5 \times 10^{20} \mathrm{~m}^{-3} \cdot \mathrm{s}$ for this TRT reactor.

This preliminary result of $n T$ indicates some (but not a large) margin of ignition that is approximately equal to that in a circular EBT reactor with SYM coils for $R_{T} \simeq 35 m[1]$.

\section{Comments on the Stability Properties of Advanced EBT}

Configurations

The maximum beta ( $\beta$ ) at which a plasma can be confined in an advanced EBT configuration is presently an unknown quantity of significant interest with respect to both the reactor embodiment of such a device and the near-term experimental devices. For low values of core plasma beta, $\beta_{c}$, and in the conventional EBT (where there is a relatively short connection between good and bad curvature regions and hot electron rings to stabilize the bad regions), the unstable modes have been expected to be close to flutelike and do not expend much energy bending field lines. For higher values of $\beta_{c}$ in closed field line devices where there is an extended region of unfavorable curvature - without rings connecting good curvature regions (such as in the square and racetrack), the stabilization due to field line bending can become smaller than the destabilization due to mode localization in the bad curvature regions, and modes localized to these regions can go unstable.

On the other hand, in devices with rotational transform and closed flux surfaces (ELMO Snakey Torus, a tokamak with energetic particles), the stability question is qualitatively different. Here an important factor is the effect of the outward shift of the flux surfaces (caused by toroidicity) and its influence on the $\int d l / B$ profile seen by the plasma. For large enough 
shifts, a stable magnetic-well configuration is formed, and arbitrarily large plasma betas can be stably confined. It has been suggested that these shifts can be initially produced by a high beta energetic particle component (hot electron ring). The only requirement on the hot species is that it remain decoupled from the core plasma and that it be present until the core plasma beta is large enough to maintain itself in the second stability regime. At this point the energetic particles would no longer be necessary and could be turned off. Finally, there are devices such as the TRT EBT with weak rotational transform which probably are closer, from the stability point of view, to the closed field line devices than to the closed flux surface device. That is, they cannot be stabilized by the outward shift (caused by a high beta plasma) moving the plasma into a region where $\int \mathrm{d} \ell / B$ is favorable, but rather must be stabilized by a self-dug well from hot electron rings. However; such a well may be easier to form in the TRT (and also in the Andreoletti coil torus) since the variation in $\int d \ell / B$ across the minor radius is typically smaller than in the conventional bumpy torus.

We first discuss stability calculations relevant to closed field line devices and then mention those for closed flux surface devices since the motivations for using an energetic particle component are somewhat different in the two cases.

For closed field line devices, simplified stability calculations have been made [2] using the Euler-Lagrange equations derived from the primitive form of the energy principle (valid for low-frequency modes). If one examines the high m limit (usually the most unstable), then the perpendicular and parallel problems decouple and stability can be analyzed on a field line-by-field line basis. This method is typically applied to the outermost field line in the equatorial plane for the 
case of bumpy square and racetrack since this is the most unstable point. This analysis has indicated that the $\beta_{c}$ limit for the corners of EBS is roughly $10 \%$ and that this limit scales in proportion to the number of sides squared in the case of bumpy polygons (racetrack, square, pentagon, etc.). Therefore, it appears that the $\beta_{c}$ limit for the corners of these devices is either comparable or is not as limiting (except possibly in the case of the racetrack) as the Van Dam-Lee $\beta_{c}$ limit in the hot electron ring region.

The closed flux surface devices can be divided into (1) those in which the outward Shafranov shift can produce a stable magnetic woll [ELMO Snakey Torus (EST) [6] and tokamak with energetic particles [9]], allowing access to the second stability regime, and (2) those in which the shift cannot (TRT [5]) and where the ring must produce a well. The amount of rotational transform present accounts for the difference between these two groups; with the former having larger transform than the latter.

For the TRT device, stability calculations have recently been initiated based on an existing high $m$ ballooning formalism. In this device localized modes that can grow in the connecting ends of the racetrack are physically separated from the stabilizing rings. Preliminary estimates based on examining the connection lengths have indicated that ballooning in the end sections does not become a problem until $\beta_{c}$ is comparable to the Van Dam-Lee ring (core ccupling $\beta_{c}$ limit in the mirror sectors).

In the EST helically displaced magnetic field coils are used to produce a device with closed magnetic surfaces [6]. The hot electron plasma is initially present to produce a sufficiently large Shafranov shift (about $10 \%$ hot electron beta required) to stabilize pressure-driven modes in the core plasma. Once the core 
plasma beta is in the $20 \%$ range, power to the hot electrons can be turned off, allowing them to decay and deposit their energy as heat into the core plasma. To achieve stability it has been estimated that a ring beta is needed that is comparable to that required to dig a well in a conventional EBT.

\section{References}

[1] N. A. Uckan et al., EBT Reactor Analysis, Oak Ridge National Laboratory Report ORNL/TM-8712, 1983.

[2] N. A. Uckan, ed., Advanced Bumpy Torus Concepts: Proceedings of the Workshop CONF-830753, Oak Ridge, Tenn., 1983, papers published therein.

[3] R. L. Miller, R. A. Dandl, and G. E. Guest, "Drift Surface Stldies of EBT Configurations with Noncircular Magnetic Coils," in Ref. [2], p. 37.

[4] L. W. Dwen, D. K. Lee, and C. L. Hedrick, "ELMO Bumpy Square," in Ref. [2],p. 55.

[5] J. F. Pipkins, R. J. Schmitt, and H. B. Ard, "Optimized Approaches to EBT's with Simple Circular Coils," in Ref. [2], p. 99.

[6] A. H. Boozer, "The ELMD Snakey Torus," in Ref. [2], p. 161.

[7] L. H. Owen and N. A. Uckan, "EBT Reactor Magnetics and Farticle Confinement." J. Fusion Energy 1,341 (1981).

[8] D. G. HcAlees et al. The ELMO Bumpy Torus Reactor (EBTR) Reference Design Dak Ridge National Laboratory Report ORNL/TM-5669, 1976.

[9] J. W. Van Dam et al., "Energetic Particles in Tokamaks: Stabilization of Ballooning Modes, "in Ref. [2], p. 167. 


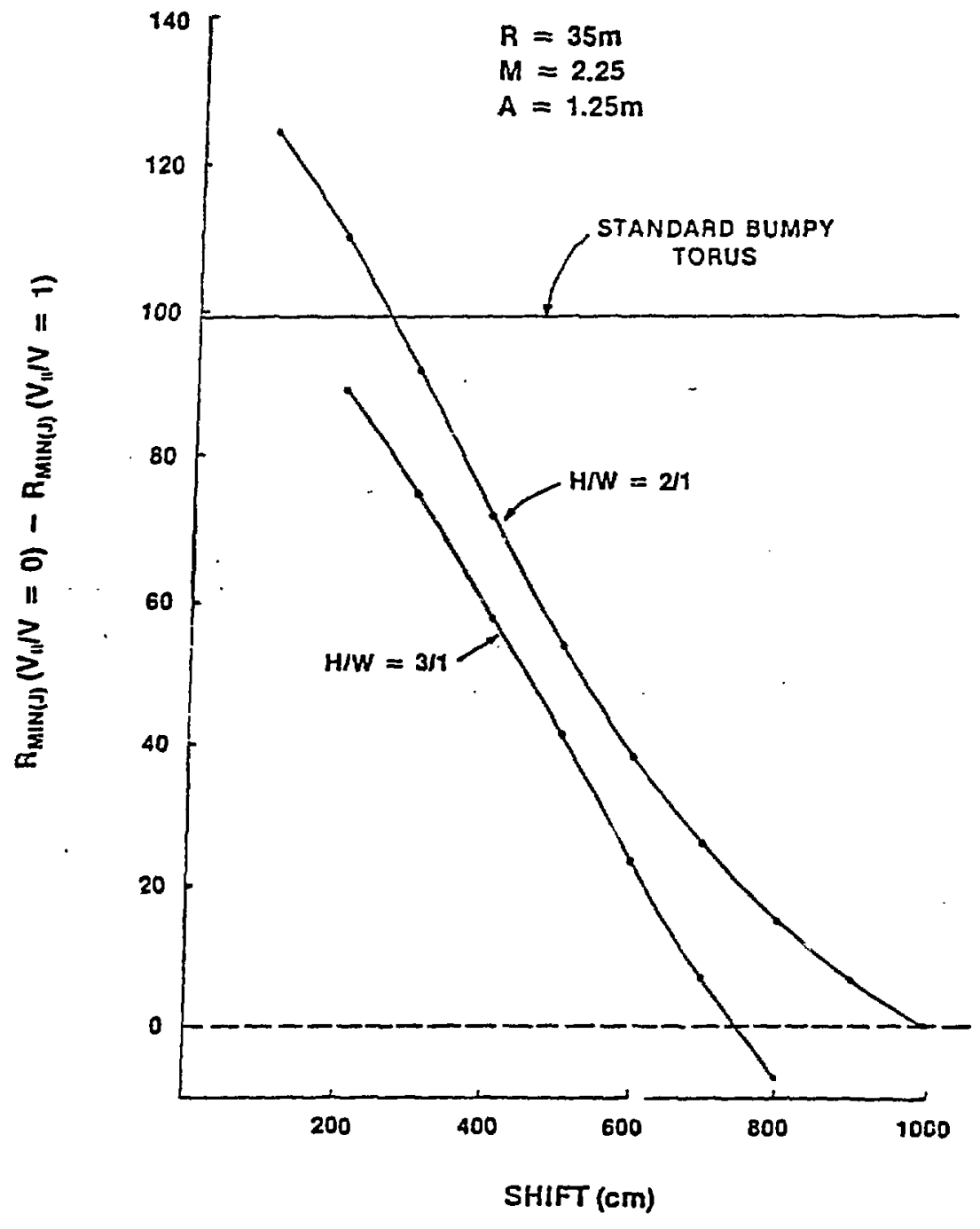

Figure 1 


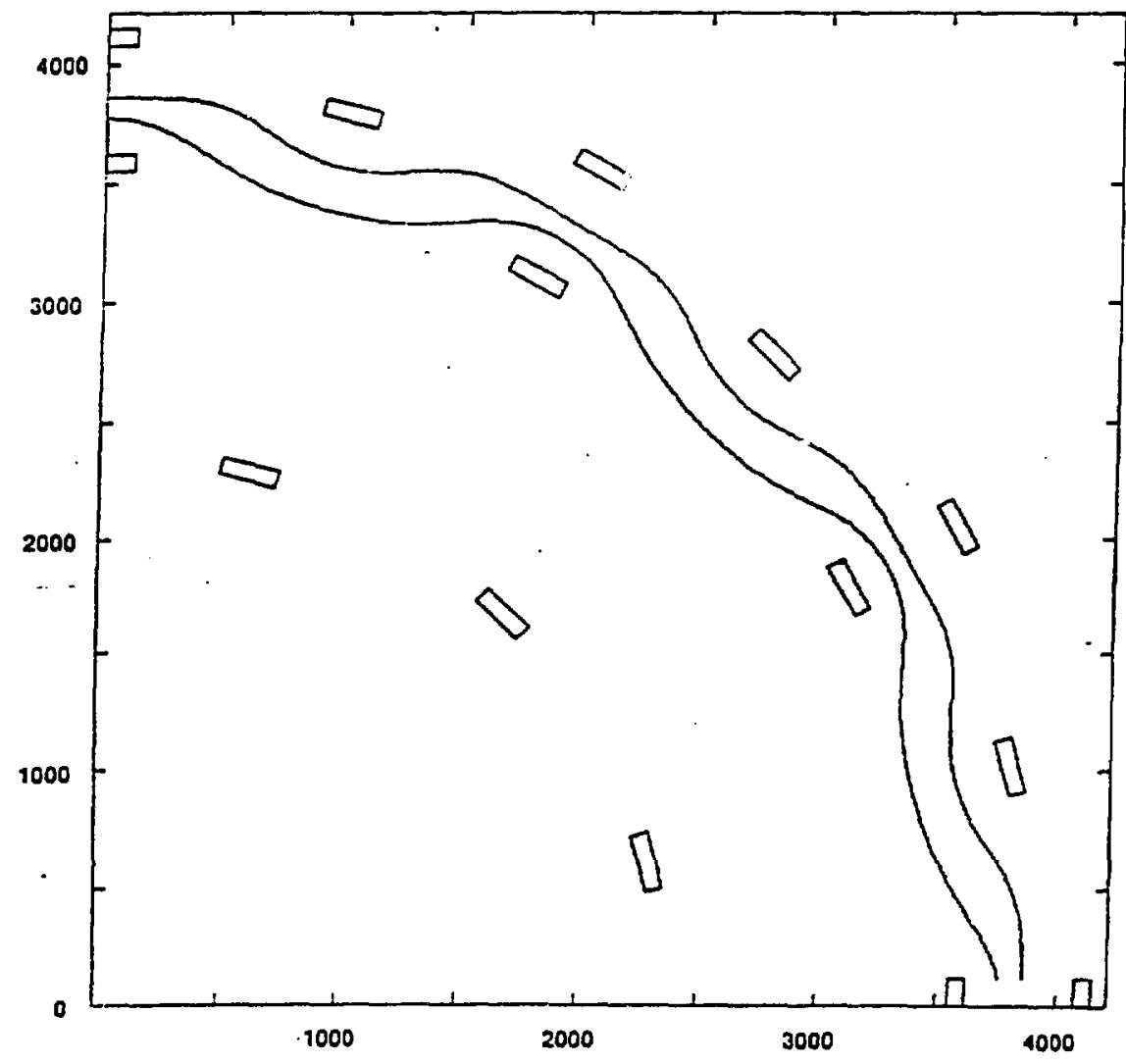

Figure 2 


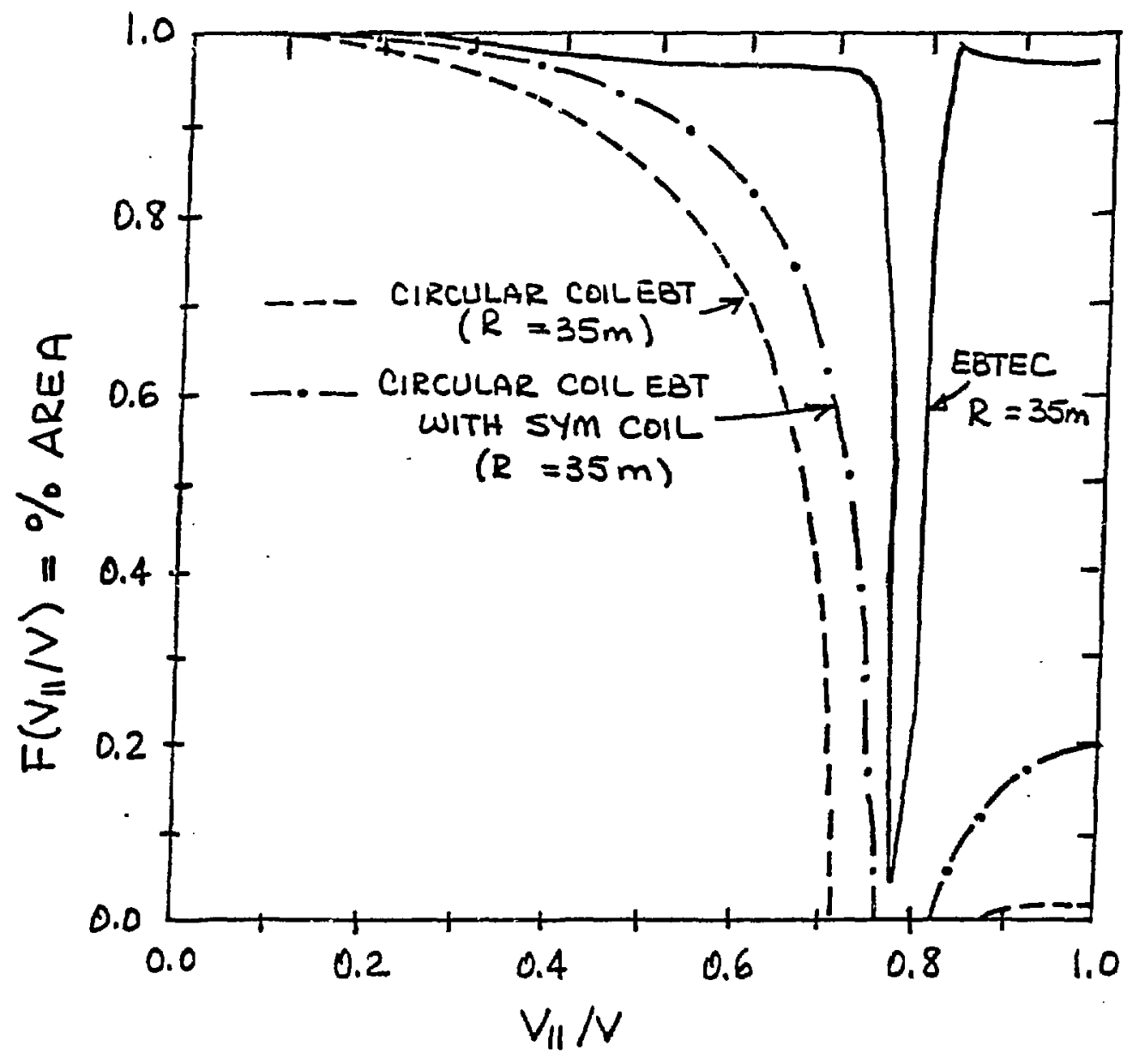

Figure 3 (a) 


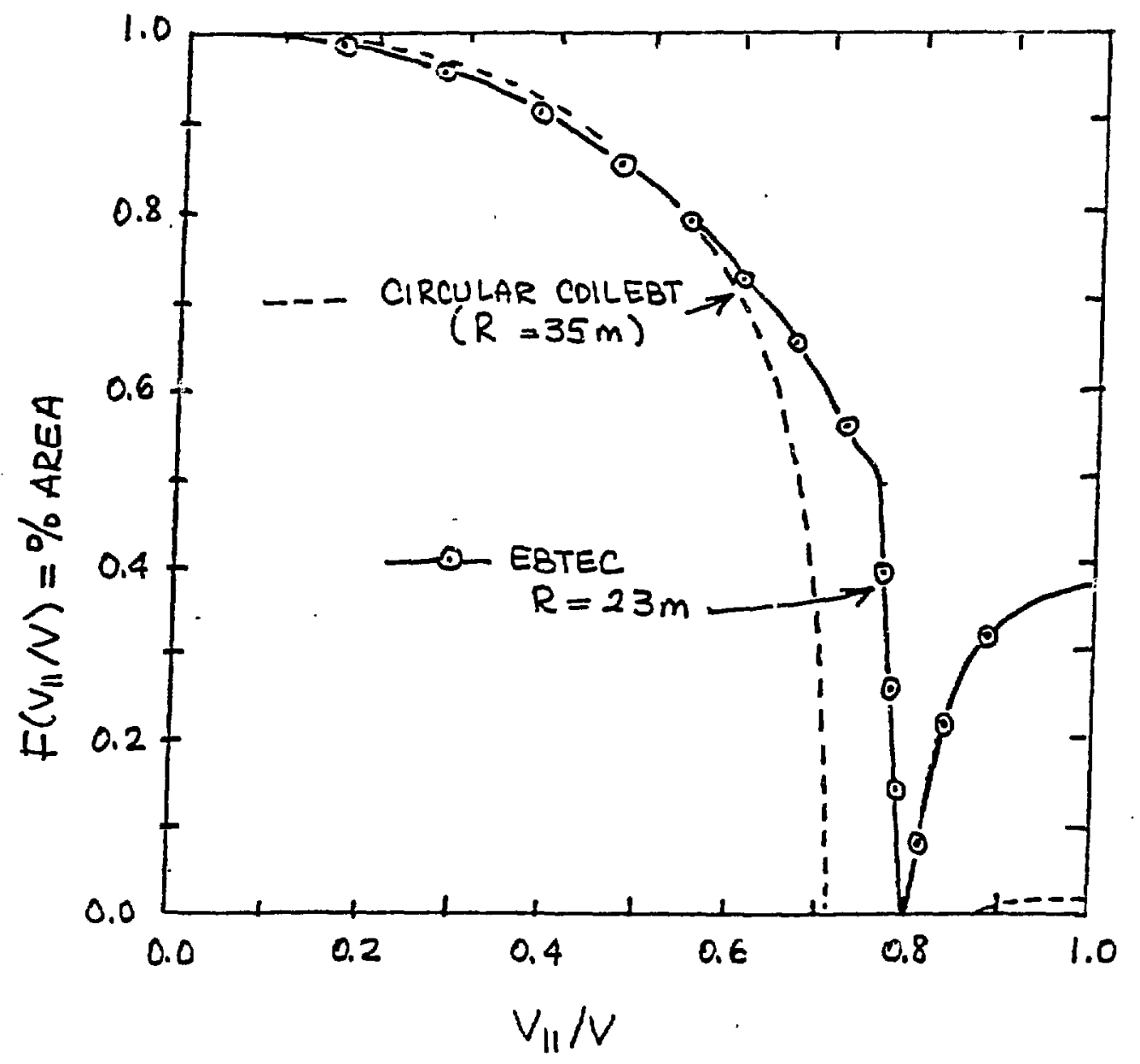

Figure $3(b)$ 


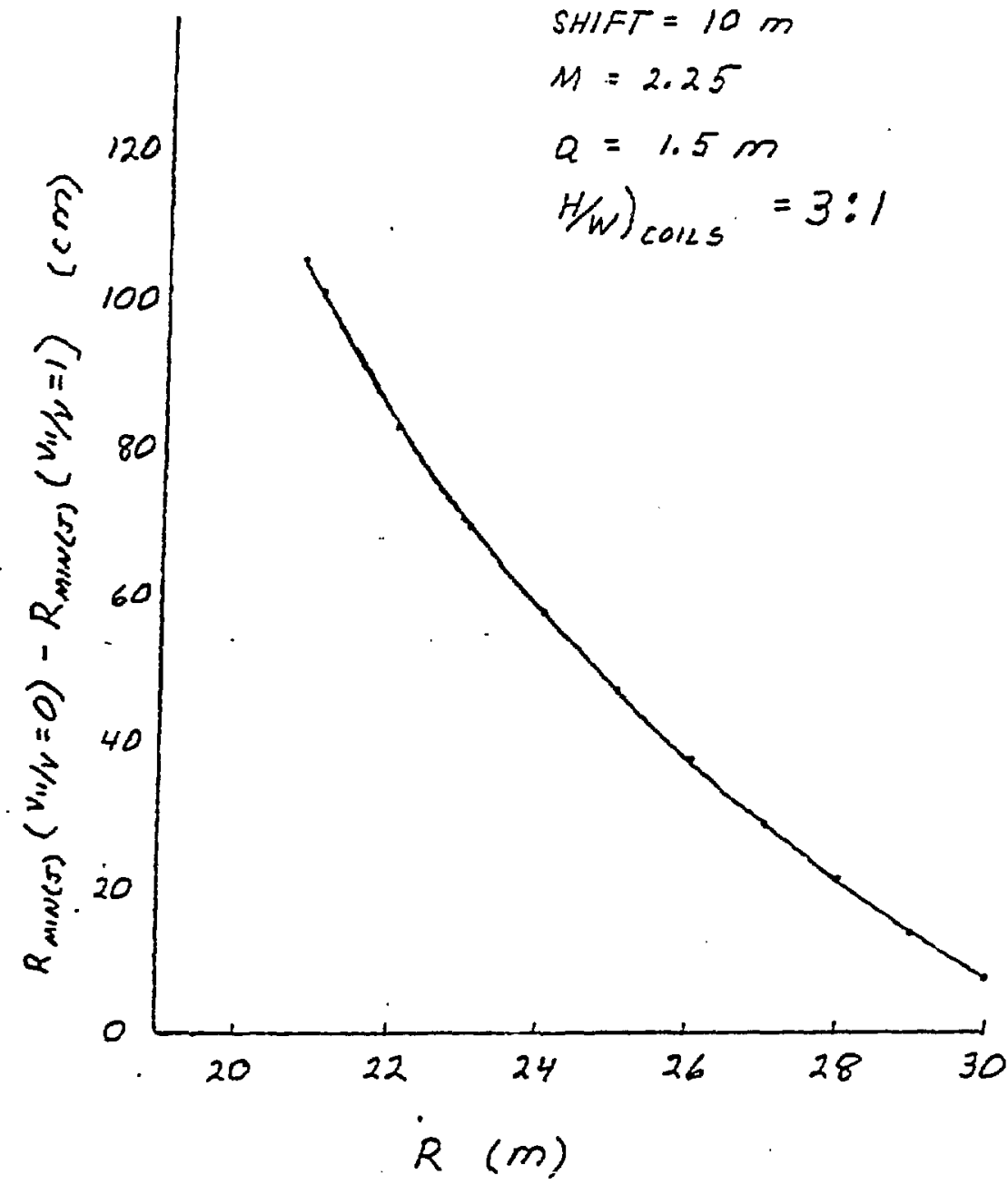

Figure 4 


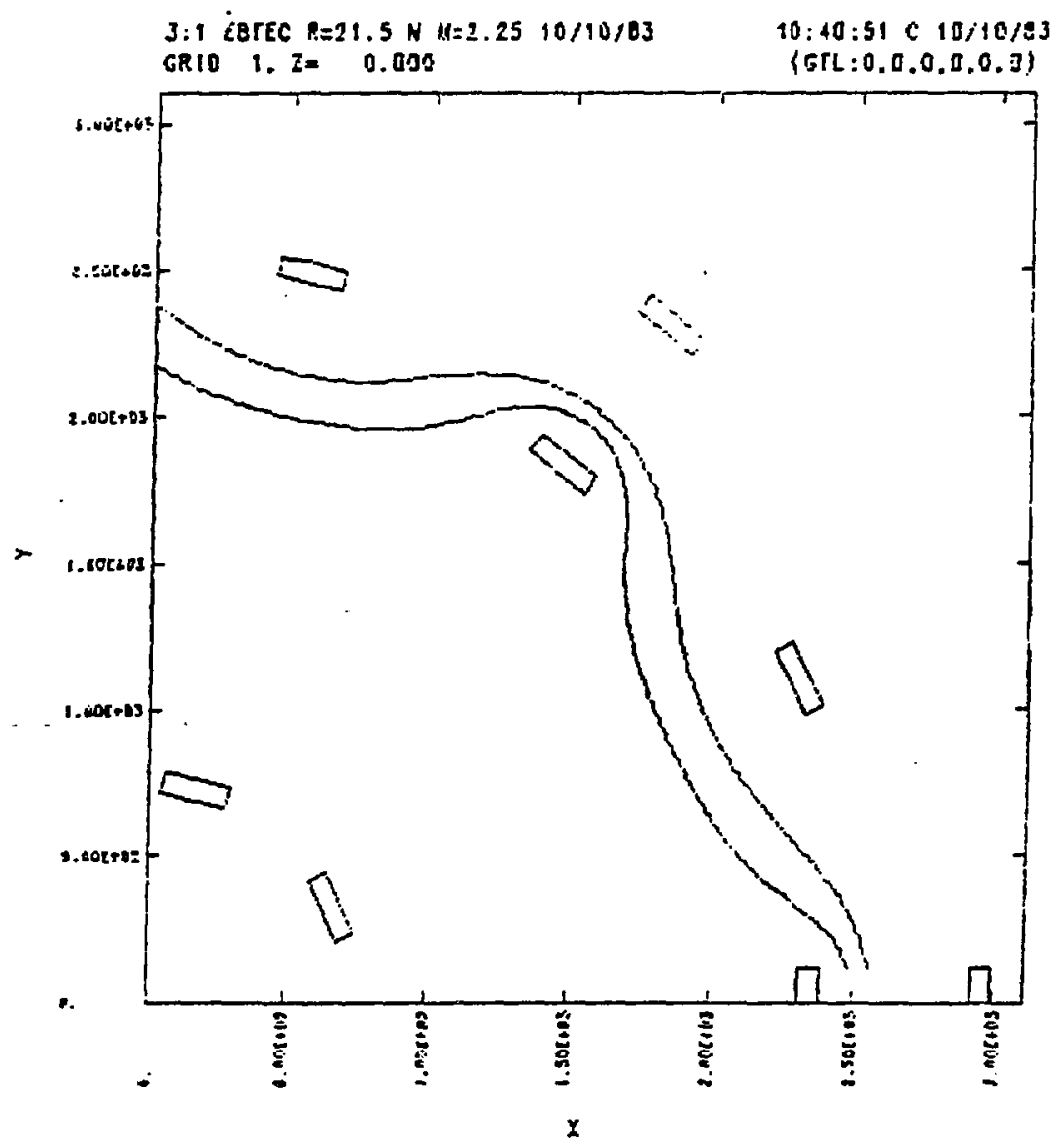

Figure 5 


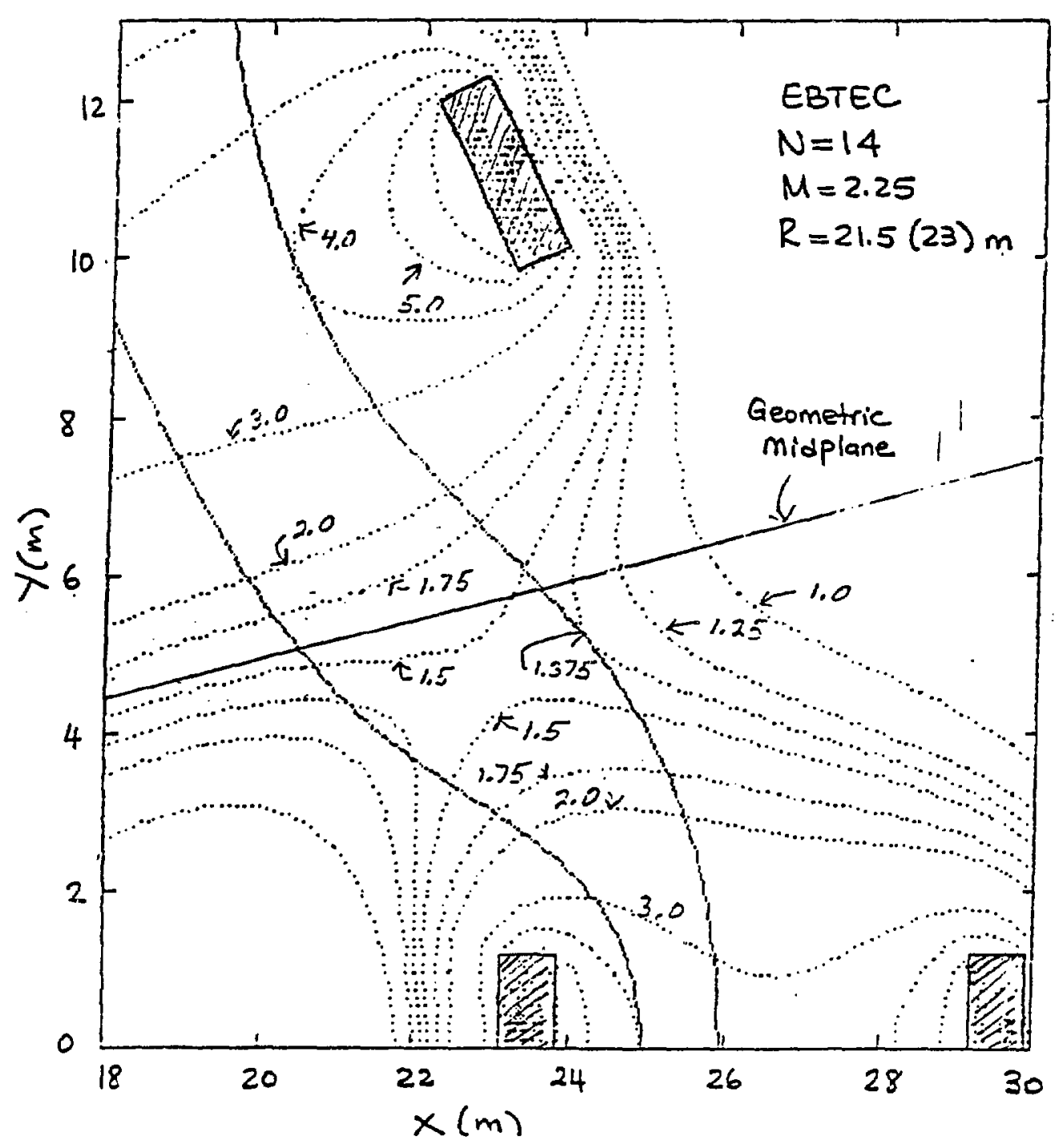

Figure 6 


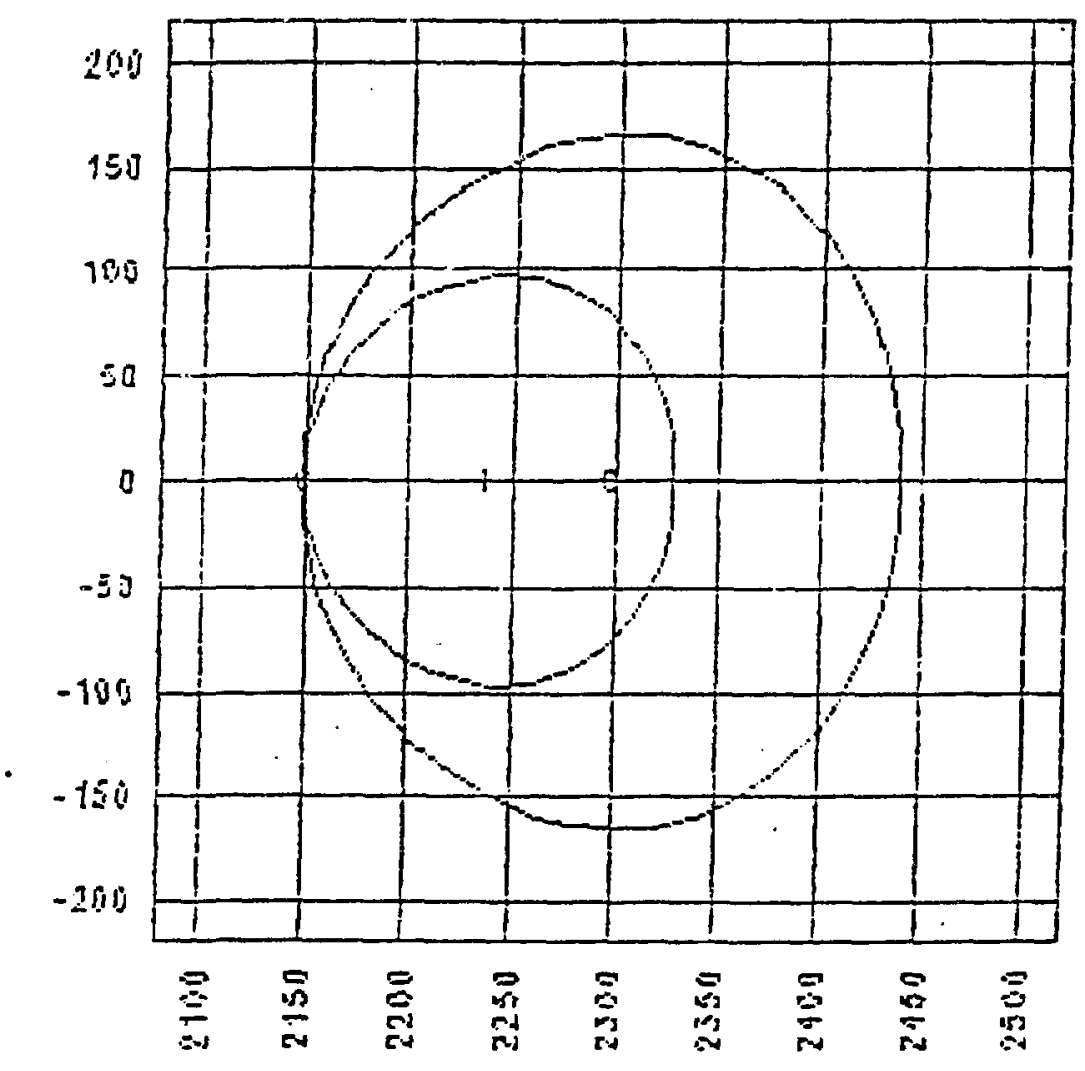

Figure 7 


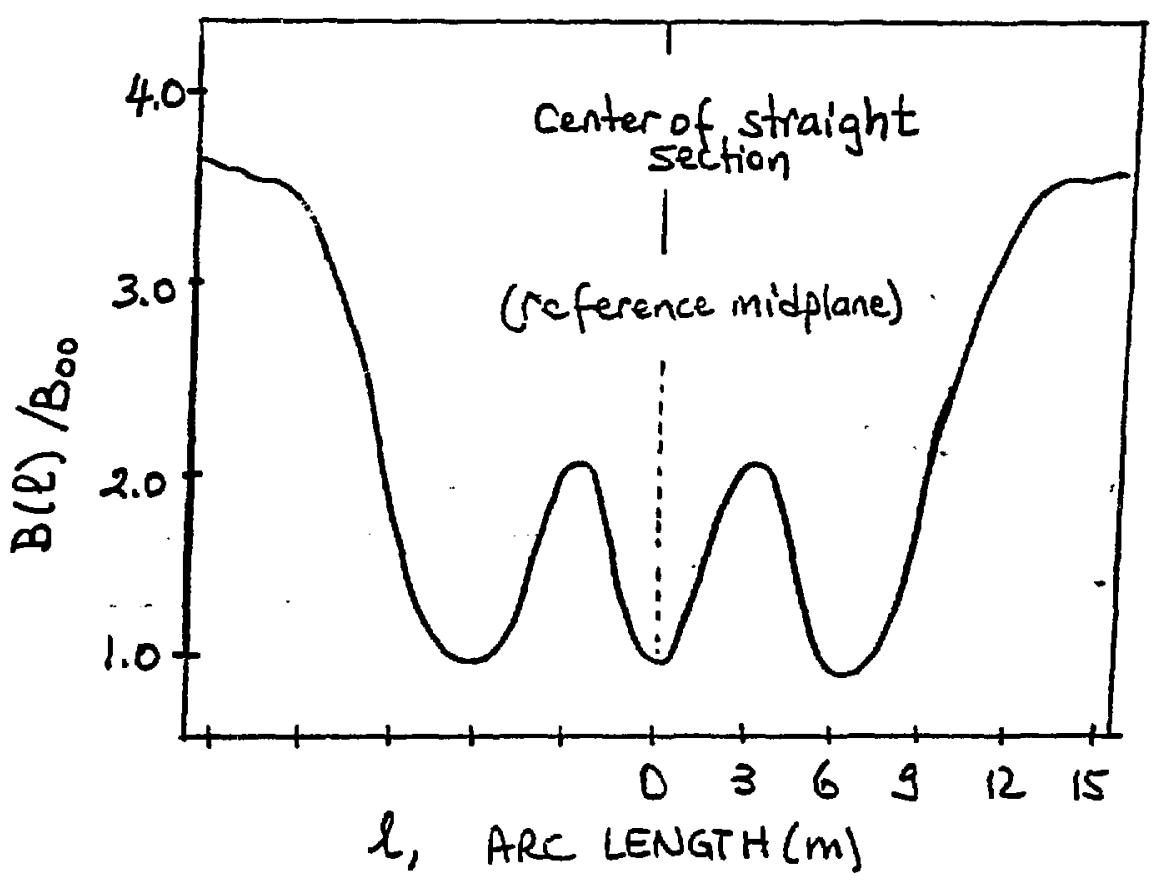

Figure 8 


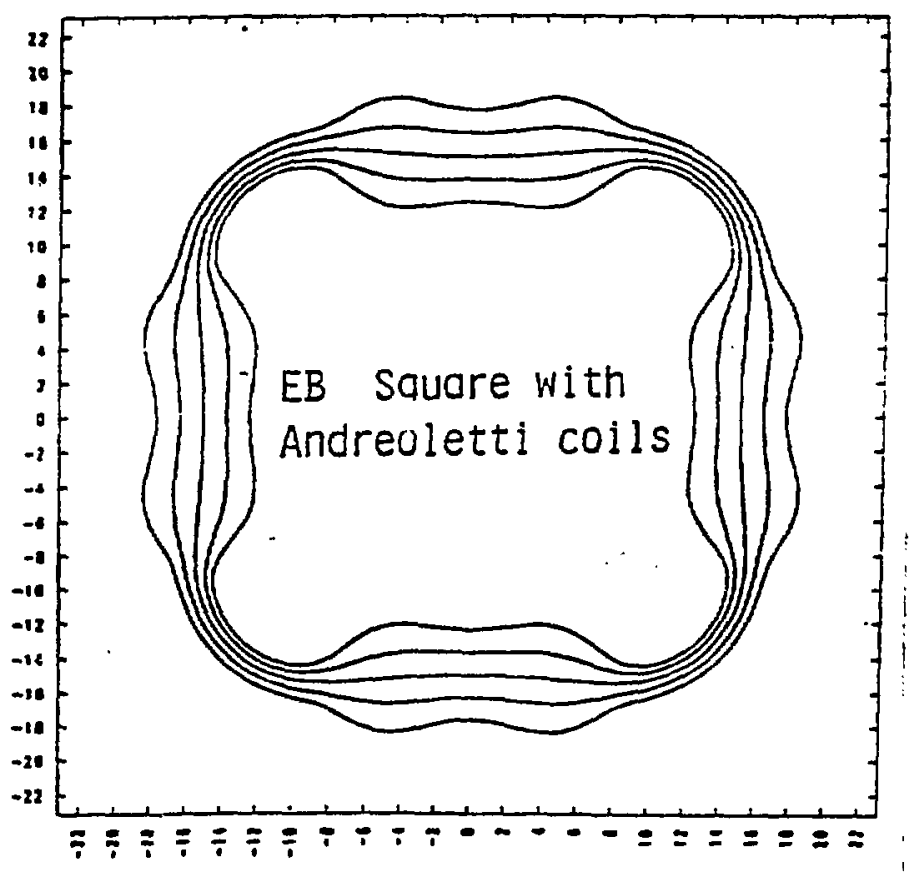

Figure 9 


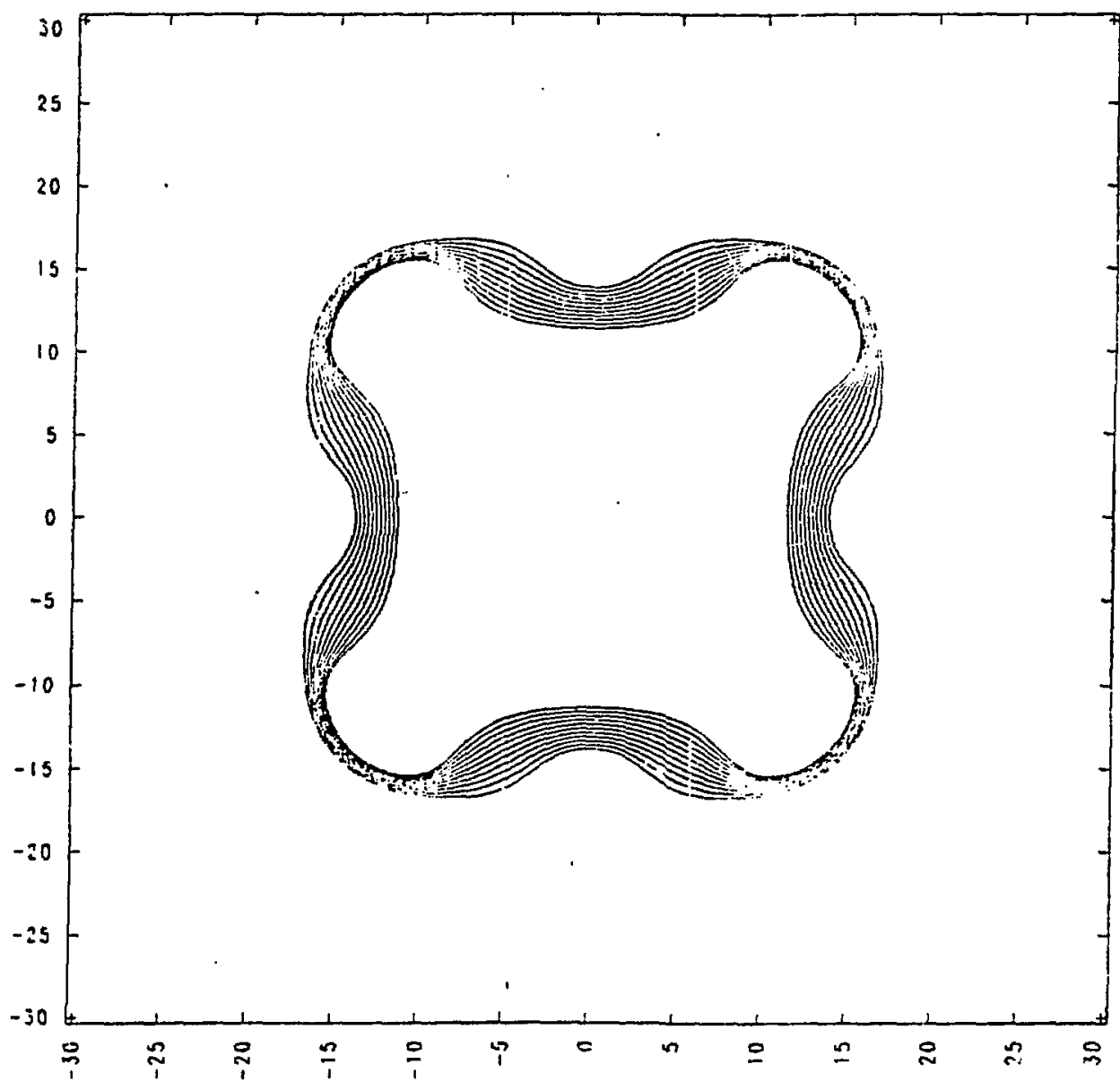

Figure 10 


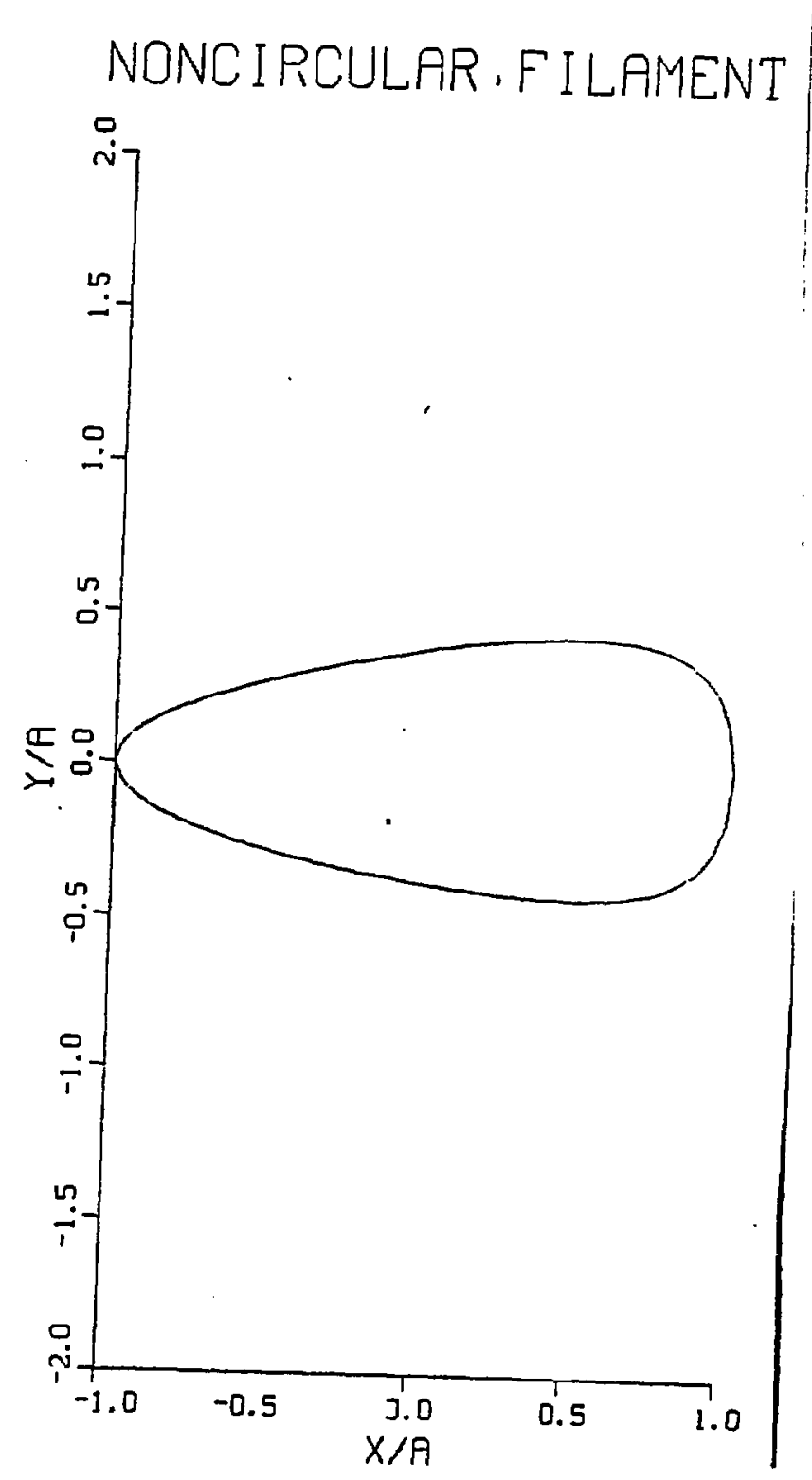

Figurell 


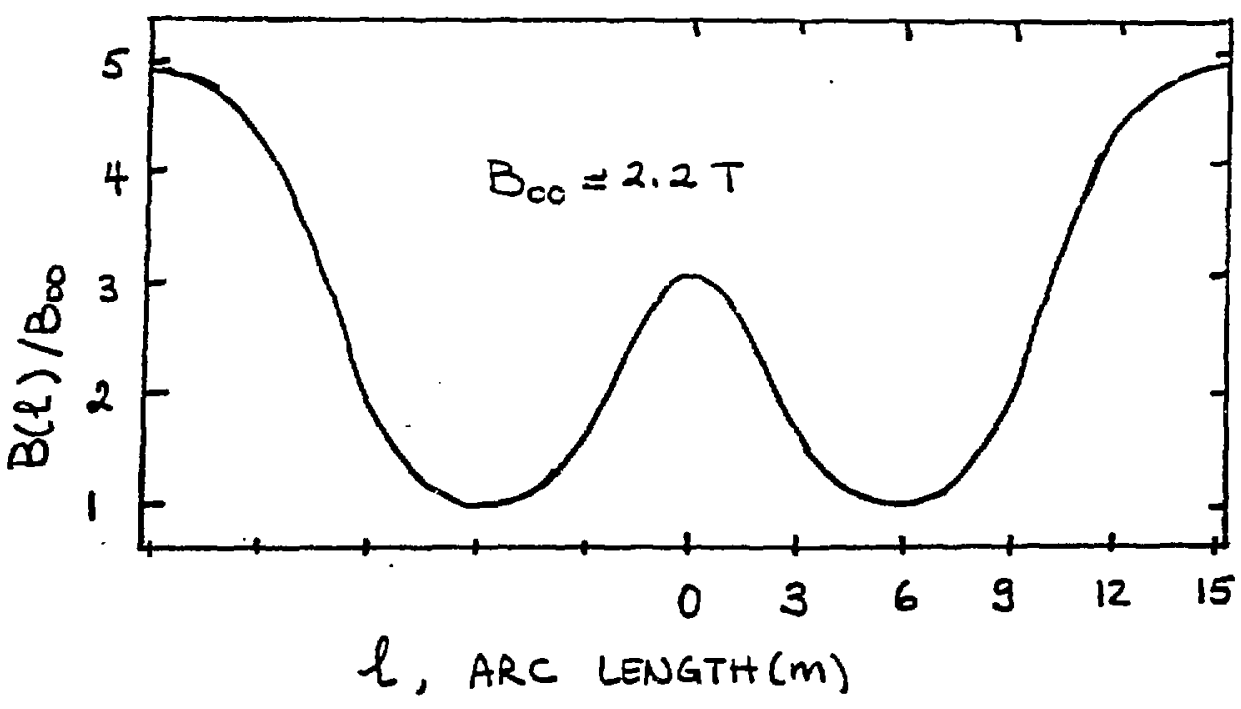

Figure 12 


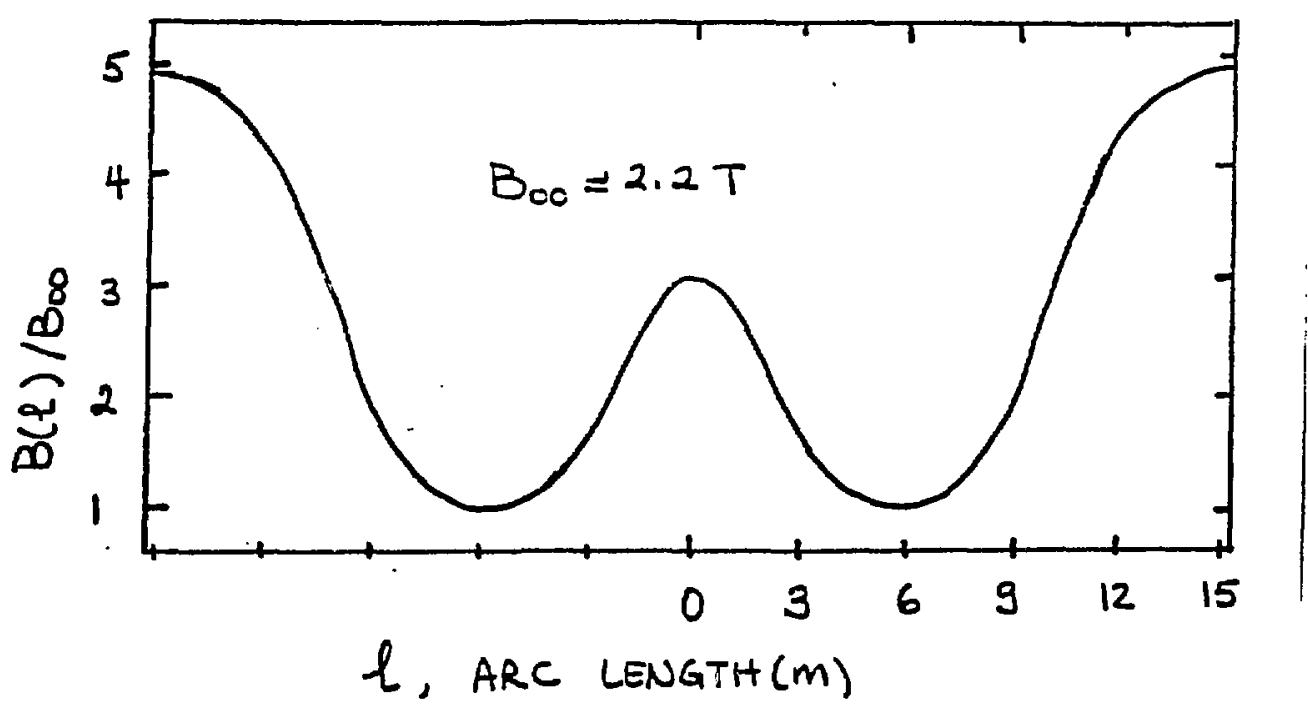

Figure 12 


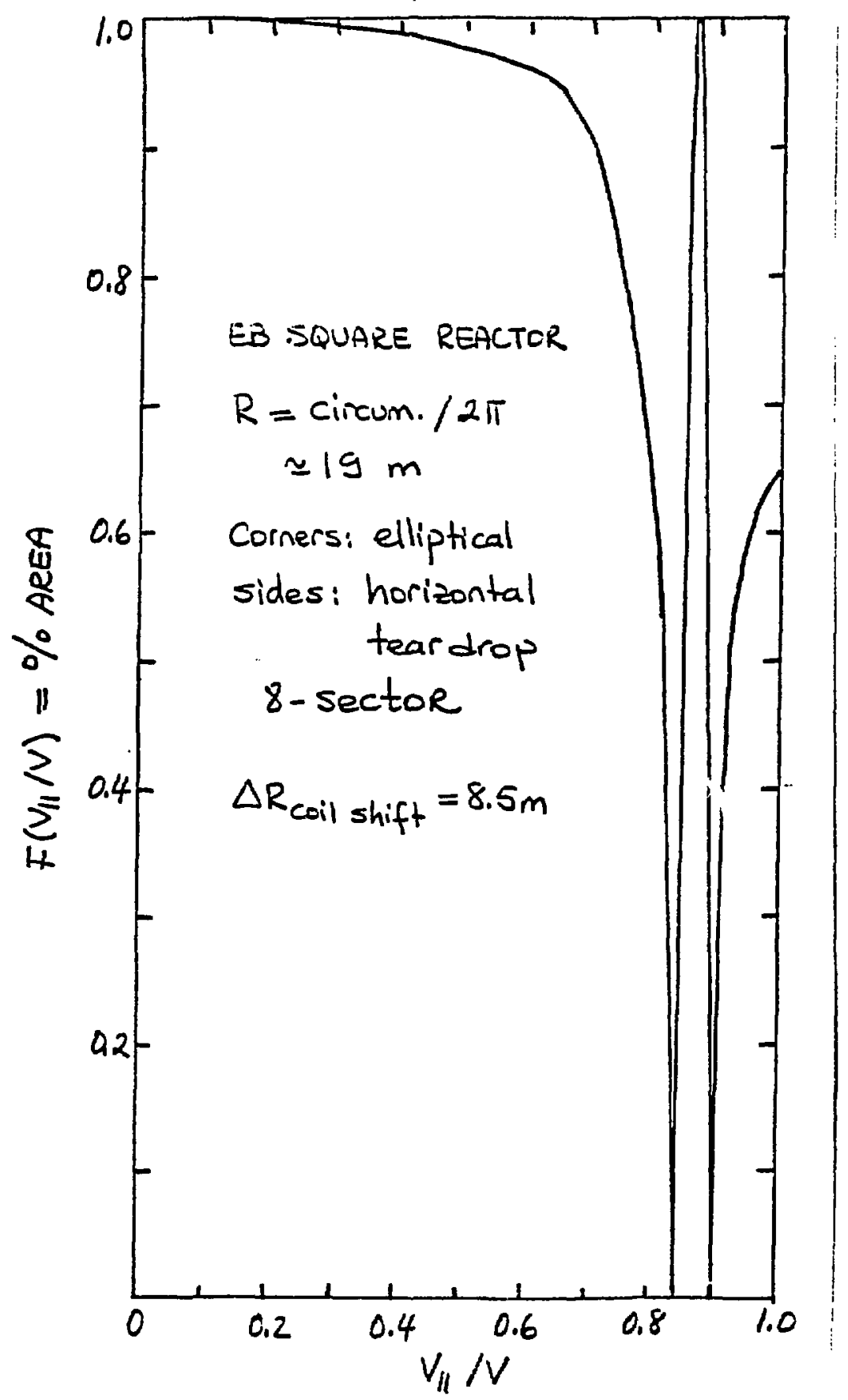

Figure 13 


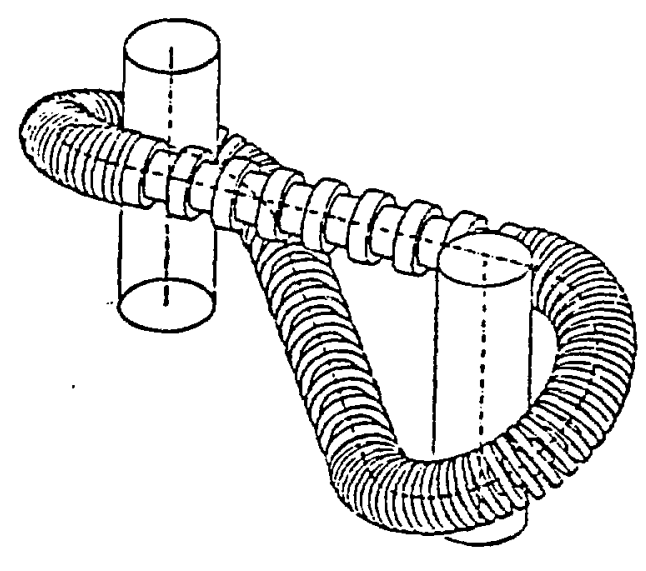

Figure 14 